\title{
Dependence of inner-shell vacancy production upon distance in hard $\mathrm{Li}$-Al collisions
}

\author{
John W. Hartman, M. H. Shapiro, ${ }^{*}$ and T. A. Tombrello \\ Department of Physics, Mathematics, and Astronomy 200-36, California Institute of Technology, Pasadena, California 91125 \\ J. A. Yarmoff \\ Department of Physics, University of California, Riverside, California 92521
}

(Received 26 August 1996)

\begin{abstract}
We match the predictions of molecular-dynamics simulations of $1.2 \mathrm{keV}$ and $2.0 \mathrm{keV}{ }^{7} \mathrm{Li}^{+}$scattered from $\mathrm{Al}(100)$ to observed total $\mathrm{Li}$ atom spectra measured by time-of-flight spectroscopy. In doing so we determine the relevant parameters in a simple distance of closest approach model for the probability of production of single and double vacancies in the $\mathrm{Li} 1 s$ shell during hard Li-Al collisions. In the standard Fano-Lichten model of vacancy production, vacancies are produced with unit probability if the collision is hard enough to force the collision partners past some critical distance of closest approach. We find that such an assumption is insufficient to fit our simulations to experimental observations, and that we must allow for a gradual turning on of the vacancy production probability as the distance of closest approach decreases. The resulting model may be useful in modeling atomic excitation effects in simulations of other ion-impact processes.
\end{abstract}

[S0163-1829(97)05007-8]

\section{INTRODUCTION}

Low-energy ion scattering from surfaces is a useful probe of surface structure and composition that integrates a number of ion-surface interactions. These interactions include inelastic losses to electronic degrees of freedom, resonant neutralization by the local electrostatic potential, charge promotion and exchange, and electron emission. Recent investigations by German et al. ${ }^{1}$ and Weare et al. ${ }^{2}$ of near $180^{\circ}$ scattering of normally incident ${ }^{7} \mathrm{Li}^{+}$ions from clean and alkali covered $\mathrm{Al}$ substrates provide a well controlled investigation of several of these ion-surface interactions. Sharp features at the high end of the scattered ion spectrum result from ${ }^{7} \mathrm{Li}^{+}$ions scattered after a single binary collision with a surface atom. Because of the large scattering angle employed, these single scattering features are well-resolved from the the multiple scattering background.

German et al. ${ }^{1}$ collected positive ion spectra resulting from ${ }^{7} \mathrm{Li}^{+} \rightarrow \mathrm{Al}$ (100) bombardment in the energy range of $0.4-5.0 \mathrm{keV}$. There are three peaks in each spectrum of scattered ${ }^{7} \mathrm{Li}^{+}$ions which, in order of decreasing energy, are labeled $P 1, P 2$, and $P 3$. The highest-energy peak, $P 1$, is attributed to elastic scattering of the ${ }^{7} \mathrm{Li}^{+}$from the target to the detector after a single binary collision with an $\mathrm{Al}$ surface atom. The peaks $P 2$ and $P 3$ are similarly explained if one assumes that a discrete energy loss also occurs during the collision.

Through kinematic considerations alone, the binarycollision model $(\mathrm{BCM})$ predicts that if a single ${ }^{7} \mathrm{Li}^{+}{ }^{27} \mathrm{Al}$ collision is responsible for scattering a lithium atom of incident energy $E_{i}$ through an angle $\phi$, then the energy of the outgoing ${ }^{7} \mathrm{Li}^{+}$atom is given by

$$
E_{f}=E_{i}\left[\frac{\cos \phi+\left(\frac{27^{2}}{7^{2}}-\sin ^{2} \phi\right)^{1 / 2}}{1+\frac{27}{7}}\right]^{2} .
$$

In a binary collision with a discrete inelastic loss, the final $\mathrm{BCM}$ energy is modified to

$$
E_{f}=E_{i}\left[\frac{\cos \phi+\left[\frac{27^{2}}{7^{2}}-\sin ^{2} \phi-\left(\frac{27^{2}}{7^{2}}+\frac{27}{7}\right) \frac{Q}{E_{i}}\right]^{1 / 2}}{1+\frac{27}{7}}\right]^{2} .
$$

Note that the resultant kinetic energy loss is not the same as the promotion energy. Also acting on the ${ }^{7} \mathrm{Li}^{+}$ion are continuous loss processes which decrease the energy of the scattered ions and broaden the peaks. Still, after accounting for the downward shift in each spectrum due to the continuous losses, the relative positions of $P 1, P 2$, and $P 3$ suggest that ions making up peaks $P 2$ and $P 3$ have suffered discrete losses of $Q 2 \simeq 60 \mathrm{eV}$ and $Q 3 \simeq 140 \mathrm{eV}$, respectively, during the $\mathrm{Li}-\mathrm{Al}$ collision responsible for their scattering.

The mechanisms responsible for the $60 \mathrm{eV}$ and $140 \mathrm{eV}$ losses are most probably single and double vacancy transfers from the $\mathrm{Al} \mathrm{Fermi} \mathrm{level} \mathrm{to} \mathrm{the} \mathrm{Li} 1 s$ and $\mathrm{Al} 2 p$ levels during hard $\mathrm{Li}$-Al collisions. ${ }^{1} \mathrm{~A}$ single vacancy production in the $\mathrm{Li}$ $1 s$ shell costs $59.1 \mathrm{eV}$, while a double vacancy production costs $142 \mathrm{eV}, 3,4$ both of which agree well with the losses required to explain $P 2$ and $P 3$ in the binary-collision model. Alternatively, the $\sim 140 \mathrm{eV}$ loss may be due to a two hole configuration in which the Li $1 s$ and $\mathrm{Al} 2 p$ levels each are left with a single vacancy for a total discrete loss of 133 $\mathrm{eV}^{5,6}$ In the standard correlation diagram of the Li-Al collision complex ${ }^{1}$ the $\mathrm{Li} 1 s$ levels are pushed up into the $\mathrm{Al}$ valence band suggesting that at close separation the $\mathrm{Li} 1 s^{0}$ configuration vacancy costs less than the $\mathrm{Li} 1 s^{1} \mathrm{Al} 2 p^{5}$ configuration. However, at large $\mathrm{Li}-\mathrm{Al}$ separation, the $\mathrm{Li} 1 s^{0}$ configuration costs $142 \mathrm{eV}$ compared to the smaller $133 \mathrm{eV}$ cost of the $\mathrm{Li} 1 s^{1} \mathrm{Al} 2 p^{5}$ configuration. Hence a crossing must occur during the separation phase of the collision; this makes either configuration possible. In fact, the latter con- 


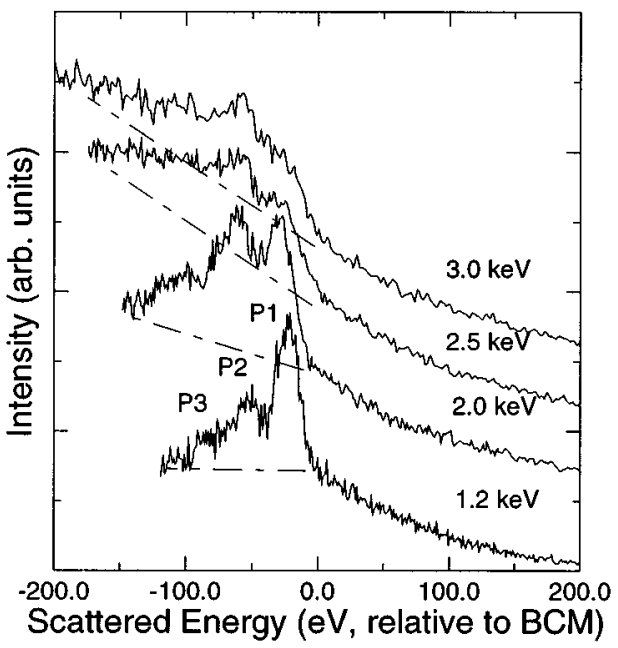

FIG. 1. Experimental time-of-flight spectra counting the total yield of $\mathrm{Li}$ scattered from $\mathrm{Al}(100)$ and detected $12^{\circ}$ from normal along the [110] direction. The detector size was $\pm 2^{\circ}$. Also indicated are our assumptions of what contribution the background of ions scattered from deeper in the target is made to each observed spectrum.

figuration is probably more likely because it represents a better equipartition of energy between the $\mathrm{Li}$ and $\mathrm{Al}$ collision partners.

We have performed molecular-dynamics (MD) simulations of the scattering of ${ }^{7} \mathrm{Li}^{+}$ions from clean $\mathrm{Al}(100)$ surfaces. For the purpose of this investigation, we modified the MD code SPUT2 (Ref. 7) to permit inelastic losses of $Q 2=59.1 \mathrm{eV}$ and $Q 3=142 \mathrm{eV}$ during "hard" Li-Al collisions. Collisions are deemed "hard" when the distance of closest approach (DCA) of the collision partners is less than some critical separation. It is important to note that MD calculations do not track the charge state of the individual atoms. As such, they predict a spectrum which includes all scattered atoms regardless of charge state.

While there is a wealth of experimentally observed positive ion spectra, ${ }^{1}$ extraction of a positive ion spectrum from MD data requires the successful integration of several effects. The probability of vacancy production during hard collisions must first be adequately described, and then the resonant neutralization of positive ions, and autoionization of excited neutrals leaving the surface must be taken into account. For $\mathrm{Li}^{+}$scattered elastically from clean Al, the fraction reaching the detector without suffering neutralization is qualitatively understood: the probability that the unexcited $\mathrm{Li}$ escapes without neutralization decreases exponentially with the time it spends near the surface ${ }^{8}$ (for a more detailed model applied to alkali systems, see Ref. 9). For inelastically scattered Li the situation is less clear. Auger and autoionization processes may fill the vacancies present in the excited $\mathrm{Li}$ during the time the $\mathrm{Li}$ is leaving the surface. When the occupation of the inner-shell changes, so does the equilibrium charge state of the $\mathrm{Li}$ atom above the $\mathrm{Al}$ surface. At present we do not have a model which unambiguously convolves these processes.

We can, however, avoid the problems inherent in modeling positive ion spectra by considering total $\mathrm{Li}$ spectra measured by time-of-flight (TOF) spectroscopy. ${ }^{2}$ Given the TOF

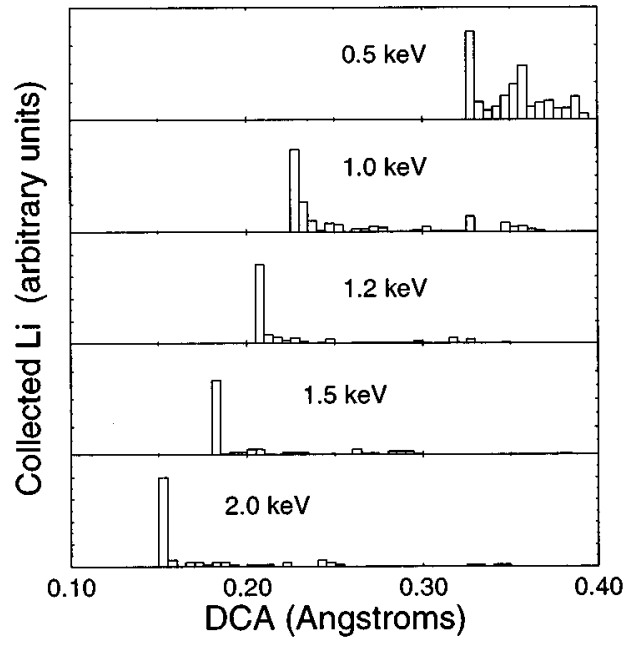

FIG. 2. Distribution of DCA's for scattering into a $\pm 10^{\circ}$ detector placed $12^{\circ}$ from normal along the [110] axis of the Al (100) surface. Results of previous, unpublished simulations of $0.5,1.0$, and $1.5 \mathrm{keV}$ are included with the presently discussed 1.2 and 2.0 $\mathrm{keV}$ simulations. Note the sharp peaking of the higher energy distributions about a single value of the DCA.

spectra, as shown in Fig. 1, the only effect we must model to match the simulation to experimental data is the probability of vacancy production during hard $\mathrm{Li}-\mathrm{Al}$ collisions.

The typical model of atomic excitation in MD calculations calls for excitation when the DCA between collision partners is less than some critical radius. In the standard Fano-Lichten model, ${ }^{10}$ Fermi pressure ejects electrons when two, roughly degenerate, orbitals are forced close together. The critical collision partner separation at which this occurs is taken to be the sum of the maximal-charge radii of the two orbitals. In Li-Al collisions the $\mathrm{Al} 2 p$ electrons, bound with $73 \mathrm{eV}$, knock out the Li $1 s$ electrons, bound with $59 \mathrm{eV}$. The critical DCA is on the order of $r_{\mathrm{Al} 2 p}+r_{\mathrm{Li} 1 s} \simeq 0.43 \AA^{\circ} .^{5}$

By increasing the energy of the incident ${ }^{7} \mathrm{Li}^{+}$ion, we can decrease the Li's distance of closest approach to the primary surface $\mathrm{Al}$ from which it is scattered. Figure 2 shows that (in the simulation) the distributions of DCA's are narrowly peaked. Thus the Fano-Lichten model would suggest that below a certain incident energy, no promotion would occur, while above it, all of the ions would be promoted. Since the experimental data strongly suggests that this is not the case, the model must be modified. We find that a gradual turning on of the vacancy production probability as the distance of closest approach decreases is required to fit our simulations to experimental observations.

\section{MOLECULAR-DYNAMICS MODEL AND PROCEDURE}

Because the interesting features in the ${ }^{7} \mathrm{Li}^{+}$spectra are due to single scattering events, our simulation targets were kept relatively small. This was necessary to keep our computation time reasonable. Unfortunately, small targets do not provide deep scattering centers responsible for the broad background signal underneath the features we wish to model. To correct for this inadequacy we are forced to assume and subtract a smooth background of multiply scattered atoms from the TOF spectra before fitting them to the simulated 
spectra. The minimal energy requirements for the TOF detector restrict the energy of the incident $\mathrm{Li}$ ion to be above $1.2 \mathrm{keV}$. Also, at high incident energies (greater than about $2.0 \mathrm{keV}$ ), the continuous losses so broaden the features that they are difficult to discern from each other. Still, we can gain valuable insight as to the nature of vacancy production in ion-surface collisions by studying this system in the range of available energies.

Our model target was prepared as a face-centered-cubic lattice of ${ }^{27} \mathrm{Al}$ with its (100) surface normal to the incident ${ }^{7} \mathrm{Li}^{+}$ion. There were five atomic layers in the target with forty-nine atoms in each layer.

All interactions were of the two-body type and derived from Moliere, $V(r)=(A / r)[0.35 \exp (-0.3 r / a)$ $+0.55 \exp (-1.2 r / a)+0.10 \exp (-6.0 r / a)], \quad$ and Morse, $V(r)=D\left\{\exp \left[-\left(r-r_{\text {eq. }}\right) / b\right]-1\right\}^{2}, \quad$ potentials. ${ }^{11}$ The ${ }^{7} \mathrm{Li}^{+}{ }_{2}{ }^{27} \mathrm{Al}$ interaction was Moliere $(A=561.561 \mathrm{eV} \AA$, $a=0.1533 \AA$ A) for all ion-atom separations. The ${ }^{27} \mathrm{Al}-{ }^{27} \mathrm{Al}$ interaction was assumed to be Moliere $(A=1946.64 \mathrm{eV} \AA$, $a=0.12547 \AA)$ for $r<1.0 \AA$, and Morse $(D=0.456 \mathrm{eV}$, $r_{\text {eq. }}=2.953 \AA, b=0.8842 \AA$ ) for $r>1.697 \AA$. The Moliere coefficient $A$ is 0.8 times the standard theoretical value $Z_{1}$ $Z_{2} e^{2}$, which permits a smoother spline between the two regions. A cubic spline, $V(r)=C_{0}+C_{1} r+C_{2} r^{2}+C_{3} r^{3}$ $\left(C_{0}=662.336 \mathrm{eV}, C_{1}=-1107.160 \mathrm{eV} / \AA, C_{2}=626.970\right.$ $\mathrm{eV} / \AA^{2}, C_{3}=-119.705 \mathrm{eV} / \AA^{3}$ ) was interpolated between the Moliere and Morse regions.

All events began with the incident ${ }^{7} \mathrm{Li}^{+}$ion $3 \AA$ above the target surface and each was integrated for fifty femtoseconds. We simulated the bombardment of the targets with normally incident ${ }^{7} \mathrm{Li}^{+}$ions of $1.2 \mathrm{keV}$ and $2.0 \mathrm{keV}$. After a coarse scan of impact parameters in the central unit cell on the surface, we determined that only those impacts within $0.42 \AA$ and $0.32 \AA$ of the surface and subsurface aluminum atoms, respectively, were scattered back from the target. This permitted us to do a much finer scan of impacts while neglecting those regions which did not contribute to the scattered ${ }^{7} \mathrm{Li}^{+}$spectrum. No attempt was made to model the effects of thermal vibrations in the lattice. At zero temperature, and for normally incident ions, the number of events to be run can be further reduced by a factor of eight by taking advantage of the symmetry of the square unit cell of the target surface. However, when using this symmetry to reduce the number of impacts run, care must be taken to count properly the ions scattered into a detector placed at an angle to the surface normal. We ran, in the central unit cell, an evenly spaced grid of impact parameters of what amounted to $1300 \times 1300$ and $1600 \times 1600$ impacts for the $1.2 \mathrm{keV}$ and $2.0 \mathrm{keV}$ incident ${ }^{7} \mathrm{Li}^{+}$ions. However, by rejecting those impacts too far from the surface or subsurface atoms to be backscattered, and by taking advantage of the surface symmetry, we were able to reduce the number of runs in each case to between 20000 and 30000 . For each impact parameter, the final position and velocity of the Li atom were saved so that its contribution to the spectrum of lithium atom energies seen at the detector could be determined.

A molecular-dynamics simulation in which the force between particles depends only on the particles' relative positions is necessarily conservative. The inclusion of discrete inelastic processes in the simulation, such as the loss due to $K$-shell vacancy production in $\mathrm{Li}$ during hard $\mathrm{Li}$-Al colli- sions, must be handled carefully. To accomplish this we use a "step-out" procedure to remove energy from binary collisions determined to be inelastic. ${ }^{7}$ When the $\mathrm{Li}$ and $\mathrm{Al}$ inelastic collision partners pass their distance of closest approach, a nonconservative integration step is taken by the integrator which moves them away from each other by a distance sufficient to decrease their relative potential energy by the amount of the discrete inelastic loss. Since the Li-Al potential is quite steep near all distances of closest approach in collisions deemed inelastic, the displacement is small. In this procedure velocities of the partners are unchanged and the step out is taken in a manner which retains their relative center of mass.

This procedure is simple and is relatively easy to introduce into an elastic molecular-dynamics code. It treats each quasibinary collision as an interaction black box: two particles enter, two particles exit, and the kinematics of the inelastic collision are satisfied. In this sense the dynamics are to be as trusted as any output from a reasonable moleculardynamics calculation - good for statistics, not good for individual trajectories. This procedure is certain to fail in cascades with high spatial density where the step-out results in a significant change in the potential energy between a binarycollision partner and some other nearby atom. In this case kinematics will not be satisfied. In the present simulations the densities are always low enough that this is not a problem.

The objective of the simulation is to find the probabilities of single and double vacancy production as a function of DCA during hard $\mathrm{Li}-\mathrm{Al}$ collisions. We have already noted (see the introduction) that even during a very hard collision, in which the $\mathrm{Li}-\mathrm{Al}$ collision partners are forced closer together than the standard Fano-Lichten radius, a vacancy is not produced with unit probability. Having the integration routine generate a random variable, calculate the probability $p_{*}$ and $p_{* *}$ for single and double vacancy production, and then perform (or not perform) the step out procedure after comparison of $p_{*}$ and $p_{* *}$ to the random variable would be an extremely inefficient method to compile statistics. First, many runs of every impact parameter which led to a possible inelastic loss would be required. Second, all of those runs would have to be repeated if new rules for $p_{*}$ and $p_{* *}$ were to be tested. To avoid this, we chose to make three parallel runs of each impact position. The first run allowed only elastic collisions; the step out was never performed. Respectively, the second and third runs performed step-out procedures corresponding to $59.1 \mathrm{eV}$ and $142 \mathrm{eV}$ losses for the first Li-Al binary collision with a DCA $<0.44 \AA$ A. In addition to the final position and velocity, for all $\mathrm{Li}$ suffering an inelastic loss, the DCA to the exciting $\mathrm{Al}$ atom was also saved. The trajectories and DCA's for all three possible vacancy production outcomes for each impact position can then be combined to allow us to predict the TOF spectra given a variety of models for the probabilities of vacancy production.

\section{RESULTS}

The experimental data shown in Fig. 1 were taken with the detector placed $12^{\circ}$ from normal along the [100] direction of the Al (100) surface. The detector acceptance was $\pm 2^{\circ}$; further details of the experiment are described in Ref. 


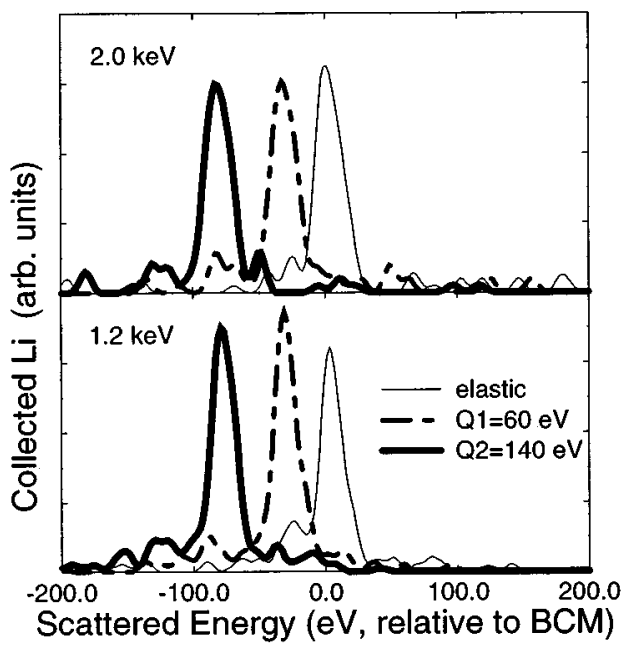

FIG. 3. Spectra of scattered $\mathrm{Li}$ atoms from simulations permitting inelastic losses of $0 \mathrm{eV}, 59.1 \mathrm{eV}$, and $142 \mathrm{eV}$ during the first Li-Al collision with a distance of closest approach of less than 0.44 $\AA$. In each case the spectrum of atoms was collected by an imaginary detector placed $12^{\circ}$ from normal along the [100] direction of the target; detector acceptance was $\pm 10^{\circ}$. The spectra have been smoothed by convolving a $5 \mathrm{eV}$ wide Gaussian with the energy of each collected $\mathrm{Li}$ atom. Note that the weight of the scattering peak is roughly unchanged even though the energy of the peak maximum decreases with increasing inelastic loss.

9. In the simulation all $\mathrm{Li}$ atoms with velocities directed into the cone of angle $\delta$ about the same detector position were collected. To improve statistics in the simulation we chose a larger detector size of $\delta= \pm 10^{\circ}$.

The simulated spectra of lithium atoms scattered elastically and inelastically in the three types of runs described above are shown in Fig. 3. In the simulation, the probabilities for single and double vacancy production and the spectra of elastically and inelastically scattered Li determine the spectrum of $\mathrm{Li}$ atoms seen at the detector. To derive the spectrum which is predicted for a particular choice of the probabilities of single and double vacancy productions, $p_{*}$ and $p_{* *}$, the following procedure is followed. For each impact position, (1) if the Li could have suffered an inelastic loss (i.e., if the DCA to any $\mathrm{Al}$ atom was less than $0.44 \AA$ ) then (a) The $\mathrm{Li}$ atom from the run which did not permit inelastic losses is checked to see if it is caught by the detector. If so, the weight $p_{0} \equiv 1-p_{*}(\mathrm{DCA})-p_{* *}(\mathrm{DCA})$ is added to the appropriate energy bin. (b) The Li atom from the run which enforced a $59.1 \mathrm{eV}$ loss for all DCA $<0.44 \AA$ is checked to see if it is caught by the detector. If so, the weight $p_{*}(\mathrm{DCA})$ is added to the appropriate energy bin. (c) The Li atom from the run which enforced a $142 \mathrm{eV}$ loss for all DCA $<0.44 \AA$ is checked to see if it is caught by the detector. If so, the weight $p_{* *}(\mathrm{DCA})$ is added to the appropriate energy bin. (2) If the Li could not have suffered an inelastic loss (i.e., if the DCA to no $\mathrm{Al}$ atom was less than $0.44 \AA$ ) then the results of all three runs are the same and the weight 1 is added to the appropriate energy bin.

To fit the simulation (performed with thin targets) to the experimental data, we must estimate the contribution of deeply scattered ions to each experimental spectrum and remove it. We assume that any sharp or sudden changes in the experimental spectra are due to the three single scattering peaks of interest, and also, that the peaks ride on the (presumably) smooth background we wish to subtract. In Fig. 1 we have indicated the background we have removed before fitting simulation to experiment. The $1.2 \mathrm{keV}$ and $2.0 \mathrm{keV}$ TOF spectra have the sharpest distinction between binarycollision features and the deep scattering background (see Fig. 1). Because of this, we have only attempted to simulate these two spectra.

We can now determine $p_{*}$ and $p_{* *}$ as a function of DCA. During the simulation, the distance of closest approach of each Li to its primary $\mathrm{Al}$ hard-collision partner (the $\mathrm{Al}$ responsible for scattering it back to the detector) was recorded. In Fig. 2 we show the distribution of primary DCA's of trajectories that result in successful scattering into the detector. [During a previous, unpublished, investigation we performed simulations of $0.5 \mathrm{keV}, 1.0 \mathrm{keV}$, and $1.5 \mathrm{keV}$ $\mathrm{Li}^{+} \rightarrow \mathrm{Al}$ (100).] For incident energies greater than or on the order of $1.0 \mathrm{keV}$ with trajectories resulting in detection, we find the distributions of DCA's to be narrowly peaked. For the $1.2 \mathrm{keV} \mathrm{Li}$ atoms scattered to the detector, the peak is centered on $0.20 \AA$, while for the $2.0 \mathrm{keV} \mathrm{Li}$ atoms, the peak is centered on $0.15 \AA$. Further, note in Fig. 3 that the effect of a discrete inelastic loss is merely a shift downward in energy of the entire peak; the weight of the peak is not appreciably changed. Given these two observations, the weights of the experimental peaks (after background subtraction) will be roughly in the ratio $W 1: W 2: W 3$ $\simeq p_{0}(\mathrm{DCA}): p_{*}(\mathrm{DCA}): p_{* *}(\mathrm{DCA})$.

After subtracting a smooth background from the experimental spectra (see Figs. 1 and 4), and using the DCA for the given incident energies found from the simulations, we see from the $2.0 \mathrm{keV}$ TOF spectrum that for DCA $\simeq 0.15 \AA, p_{0}: p_{*}: p_{* *} \simeq 0.48: 0.38: 0.14$, while from the $1.2 \mathrm{keV}$ TOF spectrum that for $\mathrm{DCA} \simeq 0.20 \AA, p_{0}: p_{*}: p_{* *}$ $\simeq 0.65: 0.29: 0.06$. Using these probabilities, and assuming a linear dependence of the vacancy production probabilities on the DCA for DCA $<0.15 \AA$, we apply the procedure described above to derive the spectra predicted by the simulations. Because of the uncertainty of the background subtraction procedure applied to the experimental spectra, sophisticated optimization criteria for evaluating the fit of simulation to the experiment were deemed unjustified. In Fig. 4 the simulated spectra derived using the procedure above can be seen to compare favorably to the measured spectra.

We can also use what we have learned from the 1.2 and $2.0 \mathrm{keV}$ simulations to extract the vacancy production probabilities at other DCA's. Mapping the DCA as a function of incident energy, we find the power law relation DCA $\simeq\left(67 \mathrm{eV} / E_{\text {inc }}\right)^{0.549} \AA$; this, of course, is valid only for the present geometry. Because a discrete loss seems simply to shift the single scattering peak downward in energy without changing the weight of the peak, we can determine the vacancy production probabilities by placing peaks at the energies predicted by the $\mathrm{BCM}$ and then adjusting their weights until the resultant spectrum resembles the observed spectrum. The relative weights of the peaks are the vacancy production probabilities $p_{0}, p_{1}$, and $p_{2}$ at the DCA for the particular incident energy. This allows us to extrapolate vacancy production probabilities to other DCA's without having to 


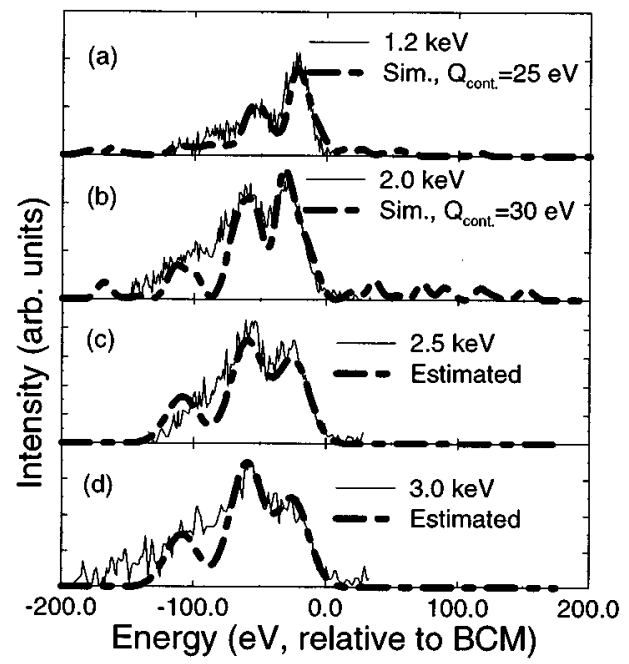

FIG. 4. TOF spectra after an assumed smooth background (see Fig. 1) has been removed. Also, in both (a) and (b), simulated elastic and inelastic spectra have been added together with weights chosen to match the experimental spectrum, the resulting simulated spectrum has also been shifted down in energy to reflect the effect of continuous losses suffered by the real ions but not modeled in the simulations. In (c) and (d), Gaussian peaks with relative separations predicted by the binary-collision model are added together with weights chosen to fit the experimental spectrum. In each case, the relative weights of the simulated results or Gaussian peaks are the probabilities of producing zero, one, or two vacancies during the scattering process.

resort to simulation. For 2.5 and $3.0 \mathrm{keV}$ incident energies, we used this method to derive the vacancy production probabilities at $\mathrm{DCA}_{2.5 \mathrm{keV}}=0.137 \AA$ and $\mathrm{DCA}_{3.0 \mathrm{keV}}=0.124 \AA$ (again, see Fig. 4). We find acceptable fits between the experimental spectra and these estimated spectra by assuming that the single vacancy production probability increases linearly with decreasing DCA and that the double vacancy production probability remains constant for DCA $<0.15 \AA$. The vacancy production probabilities versus DCA are shown in Fig. 5.

\section{DISCUSSION}

The clearest result of this study is the demonstration that the probability of vacancy production in hard collisions during ion-surface scattering turns on gradually as the distance of closest approach decreases. We note that this result is independent of whether or not we remove the uncontrolled background of scattered $\mathrm{Li}$ atoms. Our results contrast starkly with the simple Fano-Lichten model commonly used in MD simulations which assumes that the vacancy production probability has a step function dependence on the distance of closest approach.

We can understand the gradual turn on of the vacancy production probability by considering the molecular orbital model description of the colliding $\mathrm{Li}$ and $\mathrm{Al}$. When approaching the united atom limit in the molecular orbital theory of hard Li-Al collisions, the $\mathrm{Li} 1 s$ orbitals evolve toward sulfur $3 d \sigma$ orbitals, while the $\mathrm{Al} 2 p, 3 s$, and $3 p$ orbitals retain their character. ${ }^{12}$ To illustrate the excitation mechanism, assume that the ground state configuration of the

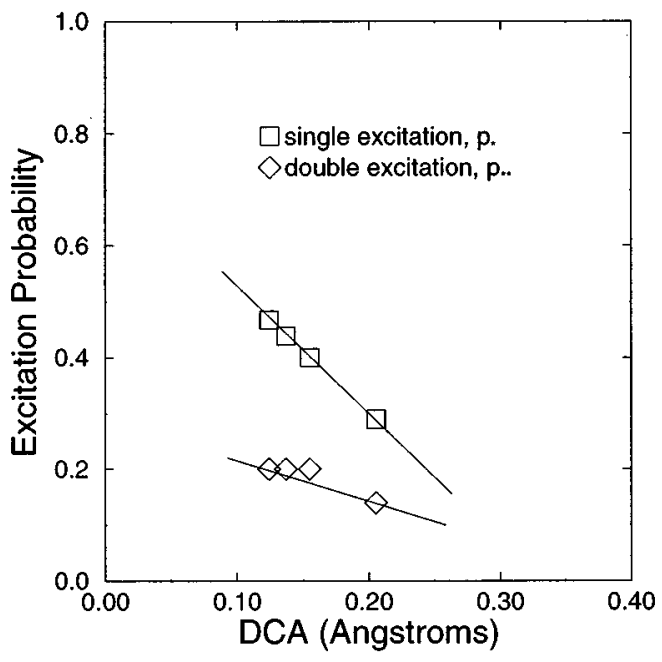

FIG. 5. Single and double vacancy production probabilities implied by the fits in Fig. 4. For the spectra estimated by summing Gaussian peaks with separations set by the BCM, the formula $\mathrm{DCA}=\left(67 \mathrm{eV} / E_{\mathrm{inc}}\right)^{0.549}$ was used to estimate the DCA for a particular incident energy in the present geometry.

$\mathrm{Al}$ valence $(n=3)$ electrons is $3 s \sigma^{2} 3 p \sigma^{1}$. Then the ground state valence $(n=3)$ configuration of the $\mathrm{Li}-\mathrm{Al}$ collision complex is $3 s \sigma^{2} 3 p \sigma^{1} 3 d \sigma^{2}$ when the Li-Al separation is large. In the united atom limit, zero Li-Al separation, the ground state configuration must be $3 s \sigma^{2} 3 p \sigma^{2} 3 p \pi^{1}$. This implies that during compression, and hence also relaxation, of the collision complex, the original configuration must become degenerate with many vacant configurations, e.g., $3 s \sigma^{2} 3 p \sigma^{2} 3 p \pi^{1}, 3 s \sigma^{2} 3 p \sigma^{1} 3 p \pi^{2}, 3 s \sigma^{2} 3 p \sigma^{1} 3 p \pi^{1} 3 d \sigma^{1}$, etc. Mixing between these configurations and the original ground state configuration is responsible for the production of $\mathrm{Li}$ inner-shell vacancies: if the final configuration has a $3 d \sigma^{2}, 3 d \sigma^{1}$, or $3 d \sigma^{0}$ occupation then zero, one, or two $\mathrm{Li}$ $1 s$ vacancies result from the collision process, respectively.

A simple model of vacancy production might assume that the various configurations are degenerate at different $\mathrm{Li}-\mathrm{Al}$ separations and occupation of the configurations after passing through the degeneracy is described by branching ratios. Then, as the Li-Al separation decreases, more degeneracies are passed providing more opportunities for the configuration to change. In this way, harder collisions provide more branching possibilities and hence a greater chance for vacancy production.

It is unclear that the valence electron wave functions are well described by the isolated molecular orbital wave functions $(3 s \sigma, 3 p \sigma$, etc.) when the collision occurs in bulk Al. In bulk $\mathrm{Al}$, the valence electrons are more like free electrons than the isolated molecular orbitals assume. However, even though the relevant wave functions and configurations may change, the spirit of the above argument should remain unchanged: At large $\mathrm{Li}-\mathrm{Al}$ separation the $\mathrm{Li} 1 s$ electrons are tightly bound beneath the Fermi sea of Al valence electrons. As the Li-Al separation decreases, the Li $1 s$-like level is pushed into the valence band becoming roughly degenerate with the $\mathrm{Al}$ valence orbitals. At this point the various local electron configurations may suffer level crossings leading to possible occupancy transfers. Harder collisions lead to more 
crossings and possible transfers and hence a greater probability of $\mathrm{Li}$ vacancy production.

We wish to note that the importance of the moleculardynamics simulations is twofold: first, they demonstrate that the weights of the scattered peaks are the same regardless of the loss imposed during the binary Li-Al collision (Fig. 4); and second, they show that the DCA's for scattering into the detector for a given incident energy are very sharply peaked (Fig. 2). These results should be easily generalizable to other systems in similar large-scattering-angle experimental configurations. Given a total atom spectrum displaying elastic and inelastic single binary scattering features $P 1, P 2$, etc., the probabilities of each of these events occurring during a binary collision are in the ratios $W 1: W 2$ : etc., where $W 1$ is the weight of the peak corresponding to the first eventuality, etc. Then, if one can assume that the distribution of DCA's is sharply peaked at some value, the probabilities of the various outcomes may be determined as a function of the incident energy. Finally, if an interaction potential is assumed and a range of incident energies are available, the probabilities may be determined as a function of distance of the closest approach, impact parameter, or other parameter of interest. In the present case we note that if we compute the DCA for a large scattering angle (i.e., $180^{\circ}$ ) using the Moliere potential, the results agrees well with those seen in the simulation for incident energies $E_{i}>1 \mathrm{keV}$. Given a better approximation to the short range interaction potential, one could easily improve the derived dependence of the excitation probabilities $p_{*}$ and $p_{* *}$ upon DCA.

We stress that the above procedure is valid only under certain assumptions. First, one must assume that a single hard scattering event is responsible both for scattering the incident ion into the detector and for causing the inelastic loss. If multiple small DCA scattering events lead to the ion finally being scattered into the detector, then one must adapt the molecular-dynamics loss procedure to deal the inelastic loss to each small DCA collision successively in separate runs of the same impact parameter. Further, one must consider the possibility that more than one inelastic loss may occur during the multiple small DCA scattering events. Obviously, the implementation of such a procedure rapidly becomes very complicated. By restricting the investigation to large scattering angles and high incident energies, one can increase the probability that only single hard collisions are responsible for scattering the ion into the detector. Given such an experimental configuration, a scan of impact parameters by a molecular-dynamics routine can then ensure that the contribution of trajectories with multiple small DCA scattering events to the total yield of backscattered ions is small.

\section{ACKNOWLEDGMENTS}

The authors wish to acknowledge helpful conversations with K. D. Shaing and Nick Choly in the early stages of this project. This work was supported in part by the Lawrence Livermore National Laboratory under subcontract B295137 of DOE Contract No. W-7405-ENG-48, and by National Science Foundation Grant No. DMR93-12468 at CSUF.
*Permanent address: Dept. of Physics, Calif. State Univ. Fullerton, CA 92634.

${ }^{1}$ K.A.H. German, C.B. Weare, and J.A. Yarmoff, Phys. Rev. Lett. 72, 3899 (1994); Phys. Rev. B 50, 14452 (1994).

${ }^{2}$ C.B. Weare, J.A. Yarmoff, and Z. Sroubek, Nucl. Instrum. Methods Phys. Res. Sect. B (to be published).

${ }^{3}$ C.E. Moore, Atomic Energy Levels, Natl. Bur. Stand. (U.S.) No. 467 (U.S. GPO, Washington, DC, 1971), Vol. 1.

${ }^{4}$ U. Bruch, G. Paul, and J. Andra, Phys. Rev. A 12, 1808 (1975).

${ }^{5}$ J.C. Slater, Quantum Theory of Atomic Structure (McGraw-Hill,
New York, 1960).

${ }^{6}$ N.W. Ashcroft and N.D. Mermin, Solid State Physics (Harcourt Brace College Publishers, Fort Worth, 1976).

${ }^{7}$ M.H. Shapiro and T.A. Tombrello, Nucl. Instrum. Methods Phys. Res. Sect. B 102, 227 (1995).

${ }^{8}$ J. Los and J.J.C. Geerlings, Phys. Rep. 190, 133 (1990).

${ }^{9}$ C.B. Weare and J.A. Yarmoff, Surf. Sci. 348, 359 (1996).

${ }^{10}$ U. Fano and W. Lichten, Phys. Rev. Lett. 14, 627 (1965).

${ }^{11}$ I.M. Torrens, Interatomic Potentials (Academic Press, New York, 1972).

${ }^{12}$ M. Barat and W. Lichten, Phys. Rev. A 6, 211 (1971). 